\title{
Unitarity of Minkowski nonlocal theories made explicit
}

\author{
Alexey S. Koshelev ${ }^{1}$ and Anna Tokareva ${ }^{2,3,4}$ \\ ${ }^{1}$ Departamento de Física, Centro de Matemática e Aplicações (CMA-UBI), \\ Universidade da Beira Interior, 6200 Covilhã, Portugal \\ ${ }^{2}$ Department of Physics, University of Jyväskylä, P.O. Box 35 (YFL), FIN-40014, Jyväskylä, Finland \\ ${ }^{3}$ Institute for Nuclear Research of Russian Academy of Sciences, 117312 Moscow, Russia \\ ${ }^{4}$ Helsinki Institute of Physics (HIP), University of Helsinki, P.O. Box 64, 00014, Helsinki, Finland
}

(Received 26 March 2021; accepted 22 June 2021; published 23 July 2021)

\begin{abstract}
In this work we explicitly show that the perturbative unitarity of analytic infinite derivative scalar field theories can be achieved using a modified prescription for computing scattering amplitudes. The crux of the new prescription is the analytic continuation of a result obtained in the Euclidean signature to the Minkowski external momenta. We intensively elaborate an example of a nonlocal $\phi^{4}$ model for various infinite derivative operators. General UV properties of amplitudes in nonlocal theories are discussed.
\end{abstract}

DOI: 10.1103/PhysRevD.104.025016

\section{INTRODUCTION}

Higher-derivative theories appear in different contexts [1-21] from pure phenomenological models to fundamental theories with the notable example of string field theory [22-25] which belongs to the particular interesting class of models featuring analytic infinite derivative (AID) operators entering Lagrangians and acting as form factors. Analyticity of these operators at low momenta guarantees a smooth local IR limit and prompts for the UV modification of respective theories.

A model example providing such a higher-derivative modification can be readily written as follows:

$$
S=\int d^{4} x\left[-\frac{1}{2} \phi\left(\square-m^{2}\right) f(\square)^{-1} \phi-\frac{\lambda}{4 !} \phi^{4}\right]
$$

where $\square$ is the d'Alembertian operator. This action is the subject of our consideration in this paper which is aimed at illustrating explicitly that the unitarity can be maintained in this class of models. The chosen model represents perhaps the simplest model featuring the properties which ought to be studied and understood. Namely, the propagator is manifestly of an infinite order in derivatives and there is an interaction term which generates a bounded from below potential. These are the features typical for nonlocal models that have applications to quantum gravity (renormalizable and ghost-free AID theories) [19] and UV finite nonlocal

Published by the American Physical Society under the terms of the Creative Commons Attribution 4.0 International license. Further distribution of this work must maintain attribution to the author(s) and the published article's title, journal citation, and DOI. Funded by SCOAP . scalar theories with an arbitrary potential $[20,26]$. It is important and interesting to perform similar computations in models more closely related to the AID gravity theories, i.e., for other spins and more general interactions, but this is beyond the scope of the current study.

Having the metric signature fixed as $(+---)$, the above Lagrangian describes a normal nonghost field provided $f=1$, which is also not a tachyon as long as $m^{2}>0$. The presence of extra derivatives may generically spoil the model by the appearance of ghosts in the spectrum. A simple way to avoid this is to demand that $f(\square)^{-1}$ be an exponent of an entire function. Then the system has no new degrees of freedom as there are no finite poles of the propagator apart from the already existing one at point $m^{2}$. This picture seems to be good especially given that the higher-derivative factor can easily provide better convergence of loop integrals.

Here, exactly a serious issue arises because an exponent of an entire function must have an essential singularity at complex infinity making the use of the Wick rotation unjustified [27]. Indeed, the latter requires that the propagator has a pole at the complex infinity, not an essential singularity, and this cannot be achieved in our model.

The situation, however, has a resolution presented in the paper by Pius and Sen [26] for the class of AID theories originating from the string field theory. The resolution consists of certain modifications to the prescription of how the loop integrals are computed. Namely, specially designed integration contours for integrals over the loop momenta are prescribed. It is interesting to note that a related idea was presented many years ago by Efimov [2]. To be phrased in short, the idea of Efimov is to perform all the inner loop integrals assuming the Euclidean signature of all the momenta. After a result is obtained, one should 
continue this result analytically for the computed amplitude to Minkowski signature for all external momenta. This procedure was claimed to gain unitary scattering amplitudes.

In the present work, using the simplest example of the fish diagram in $\phi^{4}$ theory, we illustrate that the idea by Efimov in fact leads to the same definition of the amplitude as the method elaborated by Pius and Sen. ${ }^{1}$ The latter provides rules for how to account for the poles at the complex plane when integrating over loop momenta. These rules are actually the same as in local theories, since nonlocal factors do not lead to new poles. Let us also mention that the definition of amplitudes by the formal application of the familiar Wick rotation (which is the same as doing the analytic continuation of the result upon completion of loop computations) yields a well-defined local limit. In this limit, the normal Wick rotation is recovered, as well as the unitarity of a local theory.

In our analysis, we first provide an explicit example of computations fixing $f(\square)=\exp (\alpha \square)$ that already comprises a highly nontrivial set of formulas and then move on by showing that one can integrate out internal momenta at one-loop level for an arbitrary form factor $f(\square)$. This leads us to an extensive discussion on UV properties of the amplitudes in nonlocal theories. We then end up with a conclusion and outlook.

\section{FATE OF THE WICK ROTATION}

In a local quantum field theory, the amplitudes can be defined in Minkovski space-time within the familiar rule for the poles. Then it is convenient to do a Wick rotation and go to Euclidean momenta. In nonlocal theory, however, it is often problematic to define the Minkowski amplitude because the corresponding integrals may diverge. In this section we show that there is no feasible way to choose a special nonlocal form factor which would allow for obtaining convergent Minkowski amplitudes.

Let us start with the simplest tadpole correction to the propagator depicted in Fig. 1.

Formally, in Minkowski space we can write

$$
\mathcal{A}=\int \frac{d^{4} k f\left(k_{0}^{2}-\vec{k}^{2}\right)}{k_{0}^{2}-\vec{k}^{2}-m^{2}} .
$$

Then, we can do the Wick rotation and obtain the Euclidean integral,

$$
\mathcal{A}_{E}=-i \int \frac{d^{4} k_{E} f\left(-k_{0 E}^{2}-\vec{k}^{2}\right)}{k_{0 E}^{2}+\vec{k}^{2}+m^{2}}
$$

where $k_{0 E}=-i k_{0}$. This integral can be made manifestly convergent due to an appropriate choice of the

\footnotetext{
${ }^{1}$ See also $[28,29]$.
}

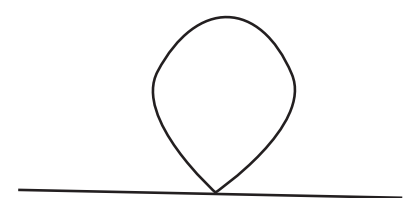

FIG. 1. Tadpole one-loop contribution to the propagator in $\phi^{4}$ theory.

function $f(\square)$. However, we cannot conclude that $\mathcal{A}=\mathcal{A}_{E}$ unless we check that the integral over an infinite arc

$$
\mathcal{A}_{C}=\int_{0}^{\frac{\pi}{2}} \frac{d^{3} k R e^{i \theta} d \theta f\left(R^{2} e^{2 i \theta}-\vec{k}^{2}\right)}{R^{2} e^{2 i \theta}-\vec{k}^{2}-m^{2}}
$$

vanishes in the limit $R \rightarrow \infty$. In this limit,

$$
\begin{aligned}
\mathcal{A}_{C}^{\infty} & =\int_{0}^{\frac{\pi}{2}} \frac{R e^{i \theta} d \theta f\left(R^{2} e^{2 i \theta}\right)}{R^{2} e^{2 i \theta}} \\
& =\int_{r}^{R} \frac{i f\left(-z^{2}\right)}{z^{2}}+\int_{r}^{R} \frac{f\left(z^{2}\right)}{z^{2}}+\int_{0}^{\frac{\pi}{2}} f(0) \frac{e^{-i \theta}}{r} d \theta \\
& =\int_{r}^{R} \frac{f(0)(i+1)-f\left(z^{2}\right)-i f\left(-z^{2}\right)}{z^{2}} .
\end{aligned}
$$

If $f(\square)=1$ then this integral is zero which makes the Wick rotation consistent in a local theory. However, in nonlocal models the situation is less obvious. If $\mathcal{A}_{C}^{\infty}=$ const $<\infty$ then $\mathcal{A}_{C}=\int \mathcal{A}_{C}^{\infty} d^{3} \vec{k}$ is divergent which means that the Minkowski amplitude is divergent too. In principle, it is possible to choose $f(\square)$ in such a way that the condition

$$
\int \frac{\left(f(0)(i+1)-f\left(z^{2}\right)-i f\left(-z^{2}\right)\right) d z}{z^{2}}=0
$$

is satisfied. However, when considering other diagrams, we obtain other conditions, finally an infinite set of similar conditions on $f(\square)$, which a priori would not be resolved simultaneously. We thus conclude that it is nearly impossible to define nonlocal amplitudes in Minkowski space in a standard way unless a mysterious combination of a form factor and the theory potential can be found, such that an infinite tower of conditions allowing the Wick rotation is resolved. Independently of the form of the nonlocal propagator, the familiar Wick rotation cannot be done. Therefore, another way to define physical nonlocal scattering amplitudes is required.

\section{UNITARITY OF THE FISH DIAGRAM}

As we have shown just before, the definition of physical amplitudes in nonlocal theories is a tricky point which most likely cannot be resolved using standard local field theory prescriptions by just making an appropriate choice of the nonlocal propagator. We thus turn to the method elaborated in papers $[2,26]$. 


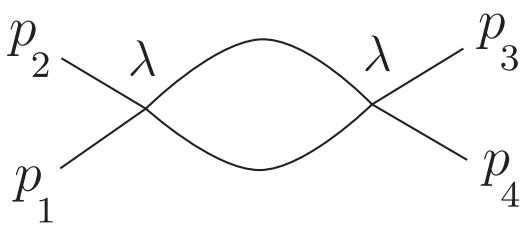

FIG. 2. One-loop contribution to $2 \rightarrow 2$ scattering in $\phi^{4}$ theory. Here only the $s$-channel contribution is shown, $p=p_{1}+p_{2}$.

We are going to study the procedure using the so-called fish diagram (See Fig. 2) in nonlocal theory (1). This diagram depends on external momenta in a combination $p=p_{1}+p_{2}$ but choosing the center of mass reference frame we can reduce the number of variables to only one scalar $p_{0}$. The corresponding Euclidean matrix element would be defined as

$$
\mathcal{M}_{E}=-\frac{\lambda^{2}}{32 \pi^{4}} I\left(p_{E}\right)
$$

The physical matrix element is to be defined as an analytic continuation of $\mathcal{M}_{E}\left(p_{E}\right)$ to Minkowski momenta $p$.

Here $I\left(p_{E}\right)$ is defined as the Euclidean integral

$$
I\left(p_{E}\right)=\int d^{4} k_{E} \frac{f\left(k_{E}^{2}\right) f\left(\left(p_{E}-k_{E}\right)^{2}\right)}{\left(k_{E}^{2}+m^{2}\right)\left(\left(p_{E}-k_{E}\right)^{2}+m^{2}\right)} .
$$

The integral can be rewritten as

$$
I\left(p_{E}\right)=2 \int \frac{f\left(k_{E}^{2}\right) f\left(\left(p_{E}-k_{E}\right)^{2}\right) d^{4} k_{E}}{p_{0 E}\left(k_{E}^{2}+m^{2}\right)\left(p_{0 E}-2 k_{0 E}\right)} .
$$

This integral has a pole at $k_{0 E}=p_{0 E} / 2$. This results in the nonzero imaginary part,

$$
\begin{aligned}
I\left(p_{E}\right)= & -\pi i \int \frac{\left(f\left(p_{0 E}^{2} / 4+\vec{k}^{2}\right)\right)^{2} d^{3} \vec{k}}{p_{0 E}\left(\vec{k}^{2}+m^{2}+p_{0 E}^{2} / 4\right)} \\
& +P . V .\left(2 \int \frac{f\left(k_{E}^{2}\right) f\left(\left(p_{E}-k_{E}\right)^{2}\right) d^{4} k_{E}}{p_{0 E}\left(k_{E}^{2}+m^{2}\right)\left(p_{0 E}-2 k_{0 E}\right)}\right)
\end{aligned}
$$

where P.V. tands for the principal value definition of the integral. Let us now continue analytically the function of $p_{E}$ to the physical momenta. Thus, we change $p_{0 E} \rightarrow-i p_{0}$,

$$
\begin{aligned}
I(p)= & -\pi \int \frac{\left(f\left(\vec{k}^{2}-p_{0}^{2} / 4\right)\right)^{2} d^{3} \vec{k}}{p_{0}\left(\vec{k}^{2}+m^{2}-p_{0}^{2} / 4-i \epsilon\right)} \\
& -2 \int \frac{f\left(k^{2}\right) f\left(\left(-i p_{0}-k_{0}\right)^{2}-\vec{k}^{2}\right) d^{4} k}{p_{0}\left(k^{2}+m^{2}\right)\left(p_{0}-2 i k_{0}\right)} .
\end{aligned}
$$

This integral is real for $p_{0}^{2}<4 m^{2}$. In the opposite case, the first term would have an imaginary part, physically corresponding to the possibility of a particle production. The corresponding expression is

$$
\operatorname{Im} I(p)=-\pi^{3} \frac{f\left(m^{2}\right)^{2} \sqrt{p_{0}^{2}-4 m^{2}}}{p_{0}} \theta\left(p_{0}^{2}-4 m^{2}\right) .
$$

Then, taking into account that nonlocal function should be normalized in such a way that $f\left(m^{2}\right)=1$ to preserve the value of the residue of the propagator, we recover the familiar result for the local theory,

$$
\operatorname{Im} \mathcal{M}=\frac{\lambda^{2}}{32 \pi} \frac{\sqrt{p_{0}^{2}-4 m^{2}}}{p_{0}} \theta\left(p_{0}^{2}-4 m^{2}\right) .
$$

It is thus obvious that one observes the canonical optical theorem statement which tells us that the imaginary part of some Feynman graph is equivalent to the product of expressions corresponding to parts in which a given graph would be cut.

We can demonstrate that the same result for the amplitude can be obtained if one takes the formal definition of nonlocal amplitude in Minkowski space,

$$
\mathcal{M}=-i \frac{\lambda^{2}}{32 \pi^{4}} I(p)
$$

Here

$I(p)=\int d^{4} k \frac{f\left(k^{2}\right) f\left((p-k)^{2}\right)}{\left(k^{2}-m^{2}+i \epsilon\right)\left((p-k)^{2}-m^{2}+i \epsilon\right)}$

and this integral over Minkowski momenta is divergent due to the inevitable presence of the essential singularity of function $f(\square)$ and consequently some growth direction at infinity as explained in the previous section. However, applying formally the Wick rotation like in a local theory we arrive to the convergent integral. This integral can be written in the following form:

$$
I(p)=-2 \int \frac{f\left(k^{2}\right) f\left(\left(p_{M}-k_{E}\right)^{2}\right) d^{4} k_{E}}{p_{0}\left(k_{E}^{2}-m^{2}+i \epsilon\right)\left(k_{0}-p_{0} / 2+i \epsilon\right)}
$$

where we have not changed $k_{0}$ to $i k_{0 E}$ in one instance to account for the pole on the real axis of $k_{0}$ properly. Namely, we first have to transform

$$
\begin{aligned}
\frac{1}{k_{0}-p_{0} / 2+i \epsilon}= & -i \pi \delta\left(k_{0}-p_{0} / 2\right) \\
& +P . V \cdot\left(\frac{1}{k_{0}-p_{0} / 2+i \epsilon}\right)
\end{aligned}
$$

and then change $k_{0} \rightarrow i k_{0 E}$ in the second term. The other pole is not pinched by the contour. After that we arrive at 


$$
\begin{aligned}
I(p)= & \pi i \int \frac{d^{3} \vec{k}\left(f\left(p_{0}^{2} / 4-\vec{k}^{2}\right)\right)^{2}}{p_{0}\left(\vec{k}^{2}+m^{2}-p_{0}^{2} / 4-i \epsilon\right)} \\
& +2 i \int \frac{f\left(k_{E}^{2}\right) f\left(\left(p-k_{E}\right)^{2}\right) d^{4} k_{E}}{p_{0}\left(p_{0}-2 i k_{0 E}\right)\left(k_{E}^{2}+m^{2}\right)}
\end{aligned}
$$

which differs from (11) by the factor $-i$ and this is precisely compensated by $i$ in the definition of amplitude (14).

Thus, the accurate definition of nonlocal amplitudes through Euclidean integrals analytically continued to Minkowski external momenta is equivalent to the familiar one used for local theories. Even though the latter cannot be formalized through the Wick rotation in nonlocal theories because the Minkowski integral is divergent, this formal approach gives the same results. Further, as it was proven in [26], the way of the amplitude's definition through Euclidean integrals preserves the unitarity of nonlocal theories and the optical theorem. Also, one can easily see that the local limit $f(\square) \rightarrow 1$ restores the standard textbook answers.

\section{FISH DIAGRAM 2-2 SCATTERING}

In this section we consider the model with A nonlocal function of the simplest form allowing for the analytic computation of the amplitude, $f(\square)=e^{\alpha \square}$. The matrix element can be formally defined as

$$
\mathcal{M}=-i \frac{\lambda^{2}}{32 \pi^{4}} I(p)
$$

where

$$
I(p)=\int d^{4} k \frac{e^{\alpha\left((p / 2+k)^{2}+(p / 2-k)^{2}\right)}}{\left((p / 2+k)^{2}-m^{2}\right)\left((p / 2-k)^{2}-m^{2}\right)} .
$$

As has been already discussed above, the Minkowski integral is divergent and the physical amplitude is defined in another way. We replace loop momenta by their Euclidean counterparts as we would do for the Wick rotation in a local theory and then obtain $I(p)$ as a sum of the Euclidean integral and the pole part,

$$
I(p)=I_{\mathcal{E}}(p)+I_{\text {pole }}(p) .
$$

The pole part here stands for the portion of the whole expression which corresponds to poles which appear at points $k_{0}= \pm p_{0} / 2 \pm \sqrt{\vec{k}^{2}+m^{2}}$ as long as these poles lie in the first and third quarter of the integration contour plane upon the prescription $m^{2} \rightarrow m^{2}-i \epsilon$.

Let us start with the computation of the Euclidean part,

$$
\begin{aligned}
I_{\mathcal{E}} & =i e^{\alpha p^{2} / 2} \int \frac{d k_{0} d^{3} k e^{-2 \alpha\left(k_{0}^{2}+\vec{k}^{2}\right)}}{\left(k_{0}^{2}+\vec{k}^{2}+m^{2}-p^{2} / 4+i p k_{0}\right)\left(k_{0}^{2}+\vec{k}^{2}+m^{2}-p^{2} / 4-i p k_{0}\right)} \\
& =2 \pi i e^{\alpha p^{2} / 2} \int \frac{d r d \theta r^{3} \sin ^{2} \theta e^{-\alpha r^{2}}}{\left(r^{2}+m^{2}-p^{2} / 4\right)^{2}+p^{2} r^{2} \cos ^{2} \theta} \\
& =\frac{4 \pi^{2} i e^{\alpha p^{2} / 2}}{p^{2}} \int r d r e^{-2 \alpha r^{2}}\left(\sqrt{\frac{\left(r^{2}+m^{2}-p^{2} / 4\right)^{2}+p^{2} r^{2}}{\left(r^{2}+m^{2}-p^{2} / 4\right)}}-1\right) .
\end{aligned}
$$

In the massless limit,

$$
\begin{aligned}
I_{\mathcal{E}}= & \frac{4 \pi^{2} i e^{\alpha p^{2} / 2}}{p^{2}}\left(\int_{0}^{p^{2} / 4-\epsilon_{0}} d z e^{-2 \alpha z} \frac{z}{p^{2} / 4-z}\right. \\
& \left.+\int_{p^{2} / 4+\epsilon_{0}}^{\infty} d z e^{-2 \alpha z} \frac{p^{2} / 4}{z-p^{2} / 4}\right) .
\end{aligned}
$$

Evaluating these integrals one obtains the contribution to $\operatorname{Re} \mathcal{M}$

$$
\begin{aligned}
I_{\mathcal{E}}^{\mathrm{Re}}= & -\frac{2 \pi^{2} i}{p^{2} \alpha}\left(e^{\alpha p^{2} / 2}-1-2 \alpha p^{2} \operatorname{Ei}\left(\alpha p^{2} / 2\right)\right. \\
& \left.+\alpha p^{2}\left(\gamma+\log \epsilon_{0}\right)\right) .
\end{aligned}
$$

Here $\gamma$ is Euler constant, $\operatorname{Ei}(z)$ is the integral exponent, and we have taken the mean value of the integrals performing the limit $\epsilon_{0} \rightarrow 0$ ). As we will see later, the divergence $\log \epsilon_{0}$ will be exactly canceled by the pole part of the amplitude in the massless limit.

The pole part is nonzero only when there are poles in I and III sectors of the complex plane, i.e., when $k^{2}+m^{2}>p^{2} / 4$. Figure 3 illustrates this situation. In this case, their impact to the amplitude is given by the sum of the relevant residues in (20)

$$
\begin{aligned}
I_{\text {pole }}= & -2 \pi i \int d^{3} k\left(\operatorname{Res}\left(k_{0}=p_{0} / 2-\sqrt{m^{2}+\vec{k}^{2}}\right)\right. \\
& \left.-\operatorname{Res}\left(k_{0}=-p_{0} / 2+\sqrt{m^{2}+\vec{k}^{2}}\right)\right)
\end{aligned}
$$

which readily simplifies to 


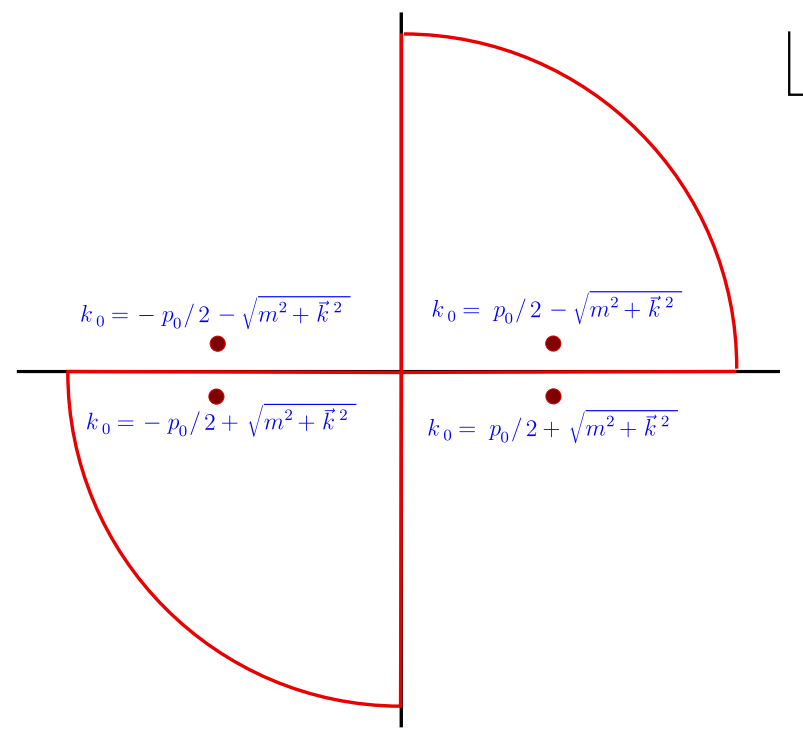

FIG. 3. Poles of the amplitude (20).

$I_{\text {pole }}=-2 \pi i \int \frac{d^{3} k e^{2 \alpha m^{2}+2 \alpha p\left(p / 2-\sqrt{\vec{k}^{2}+m^{2}}\right)}}{2 p \sqrt{m^{2}+\vec{k}^{2}}\left(p / 2-\sqrt{m^{2}+\vec{k}^{2}}\right)}$.

For $m=0$ we obtain

$$
I_{\text {pole }}=-\frac{8 \pi^{2} i e^{\alpha p^{2}}}{p^{2}} \int_{0}^{p^{2}-\epsilon_{0}} \frac{z d z e^{-4 \alpha z}}{p^{2} / 4-z} .
$$

We notice that integration up to $p^{2}$ follows from the fact that $k^{2}+m^{2}$ should be greater than $p^{2}$ in order to have poles in the I and III quarters of the integration plane.

$$
I_{\text {pole }}=\frac{2 \pi^{2} i}{\alpha p^{2}}\left(e^{\alpha p^{2}}-1-\alpha p^{2} \operatorname{Ei}\left(\alpha p^{2}\right)+\alpha p^{2}\left(\gamma+\log \epsilon_{0}\right)\right)
$$

Summing up and adding the computed in Sec. III imaginary part contribution $-\pi^{3}$ to $I(p)$ we get the final answer ${ }^{2}$

$$
\begin{aligned}
I(p)= & -\pi^{3}+\frac{2 i \pi^{2}}{\alpha p^{2}}\left(e^{\alpha p^{2}}-\alpha p^{2} \operatorname{Ei}\left(\alpha p^{2}\right)-e^{\alpha p^{2} / 2}\right. \\
& \left.+\frac{1}{2} \alpha p^{2} \operatorname{Ei}\left(\alpha p^{2} / 2\right)\right) .
\end{aligned}
$$

The real part of amplitude (19) is plotted in Fig. 4 where teh presence of a strong coupling regime seems obvious. We will see in the next sections that this situation depends on the form factor.

One can further observe, that for small arguments $\operatorname{Ei}(z) \approx \gamma+\log (z)+z$, the local limit and the logarithmic

\footnotetext{
${ }^{2}$ The real part of this amplitude was also computed in [30].
}

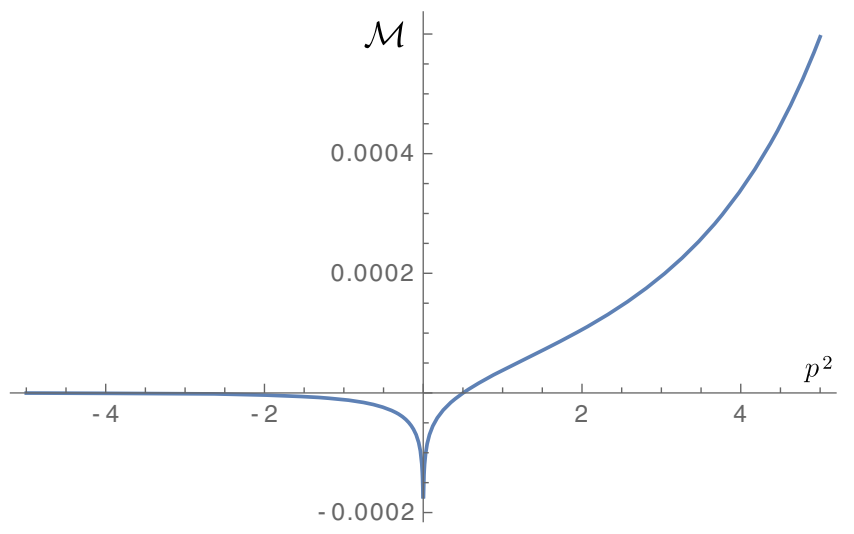

FIG. 4. Amplitude (real part) dependence on the external momentum. Here $\alpha=1, m=0, \lambda=0.1$.

singularity usually arising in the cutoff regularization restore as long as $\alpha \rightarrow 0$.

The total scattering amplitude including the crossed diagrams can be obtained as

$$
\mathcal{M}_{\mathrm{tot}}=-i \frac{\lambda^{2}}{32 \pi^{4}}(I(\sqrt{s})+I(\sqrt{t})+I(\sqrt{u}))
$$

where standard Mandelstam variables are used.

\section{COMPUTATION FOR A GENERIC PROPAGATOR}

The computation of the previous section can be extended to a generic propagator, i.e., generic form factor $f(\square)$. This can be done by utilizing the $x$ representation for propagators. This yields, in our case, the following quite simple expression for the matrix element:

$$
\begin{aligned}
\mathcal{M}(p)= & \frac{\lambda^{2}}{2(2 \pi)^{4}} \int d^{4} x \Delta(x)^{2} e^{i p x}+i \frac{\lambda^{2} \pi}{32} \\
& +\frac{\lambda^{2}}{16 p} \int_{-p / 2}^{p / 2} f(2 p q) d q
\end{aligned}
$$

where $\Delta(x)$ is the propagator in the $x$ representation and the whole formula is, in a sense, the Fourier transform of the product of the propagators. In the first term both Fourier transform and integration over $x$ are performed in the Euclidean space. The last term corresponds to the pole contribution and it has such a simple form only for even form factors $f\left(-k^{2}\right)=f\left(k^{2}\right)$. They are of a special interest because they would simultaneously decay along both real and imaginary directions in $k$.

The propagator in $x$ space can be easily written as

$$
\Delta(x)=\frac{i}{16 \pi^{4}} \int d^{4} k \frac{f\left(k^{2}\right)}{k^{2}} e^{i k x}
$$


and in contrast with a local theory, the latter integral can be straightforwardly made well defined at short distances, i.e., small $x$. First, we go to spherical coordinates and simplify the last expression as follows:

$$
\Delta(x)=\frac{i}{4 \pi} \int_{0}^{\infty} d k f\left(k^{2}\right) \frac{J_{1}(k x)}{x}
$$

where $J_{1}(z)$ is the Bessel function.

Then we get by direct substitution

$$
\mathcal{M}(p)=-\frac{\lambda^{2}}{64 \pi^{3} p} \int_{0}^{\infty} J_{1}(p x) J_{1}(k x) J_{1}(q x) f\left(k^{2}\right) f\left(q^{2}\right) d k d q d x+i \frac{\lambda^{2} \pi}{32}+\frac{\lambda^{2}}{32 p^{2}} \int_{-p^{2}}^{p^{2}} f(z) d z .
$$

One can perform an integration over $x$ in the first integral in (34) analytically and the result is as follows [31,32]:

$$
\int_{0}^{\infty} J_{1}(p x) J_{1}(k x) J_{1}(q x) d x=\left\{\begin{array}{l}
\frac{1}{2 \pi} \frac{\sqrt{\left(p^{2}-(k-q)^{2}\right)\left((k+q)^{2}-p^{2}\right)}}{p k q} \text { for }|k-q|<p<k+q \\
0 \text { otherwise. }
\end{array}\right.
$$

One further is left with an integration over $k$ and $q$ in the first integral in (34). The domain of integration can be a bit more intuitively rewritten as $\{-k+p<q<k+p$, $0<k<p\} \cup\{k-p<q<k+p, k>p\}$.

The last term in (34) demonstrates a not very much expected property. For large $p$, as long as the form factor is an integrable function, this term falls universally as $\sim 1 / p^{2}$, irrespective of a particular form factor. We will see below that for a large class of suitable functions, this makes the leading and apparently universal contribution to the amplitude.

Proceeding by computing the amplitudes numerically for different form factors $f\left(k^{2}\right)$ we choose the following two examples of a nonlocal function. As the first example we take ${ }^{3}$

$$
f_{1}\left(k^{2}\right)=e^{-k^{4}}
$$

being the simplest nonlocal function allowing for staying in the perturbative regime [33]. Besides that, we consider a more involved function which falls polynomially along the real axis while it is an exponent of an entire function. This function was first suggested by Tomboulis in [8]

$$
f_{2}\left(k^{2}\right)=e^{-\Gamma\left(0, k^{4}\right)-\gamma-\log k^{4}} .
$$

Here $\Gamma\left(0, k^{4}\right)$ is an incomplete gamma function. The latter form factor, having a polynomial decay at infinity, makes the theory avoid a possible strong coupling regime. For large real $k$, one has $f_{2}\left(k^{2}\right) \propto 1 / k^{4}$. In Fig. 5 we plot the absolute value of this function on the whole complex plane to make its growth directions visible. Also, we plot the Euclidean four-dimensional Fourier images $\Delta_{1}(x)$ and

\footnotetext{
${ }^{3}$ Hereafter in our numerical studies we set the nonlocal scale to be unity.
}

$\Delta_{2}(x)$ for the functions $f_{1}(k)$ and $f_{2}(k)$, respectively, in Fig. 6.

The resulting amplitude (real part) numerically computed with the use of (34) is plotted in Fig. 7. One can see that the curves fall for momenta much higher than the nonlocality scale. ${ }^{4}$ However, as was mentioned above, the asymptotes of this falling are of the same order $\sim 1 / p^{2}$ for both functions and are determined by the last term in expression (34). This term arises due to the pole structure in Minkowskian amplitudes. Further, one can deduce numerically that the behavior of the first term in (34) depends on the UV behavior of the nonlocal propagator and differs for the two cases under consideration. For function $f_{1}$ it is an exponential fall as $\sim e^{-p^{4}}$ while for function $f_{2}$ it is the power-law suppression $\sim p^{-6}$. We also see that the model remains weakly coupled for both chosen form factors in contrast with the $e^{\alpha p^{2}}$ form factor discussed in the previous section.

\section{UV PROPERTIES OF THE AMPLITUDES IN NONLOCAL THEORY}

The UV behavior of the scattering amplitudes is determined by the form of the nonlocal propagator. As we obtained in Sec. IV, for the propagator $e^{\square / \Lambda^{2}} / \square$ the amplitude is growing exponentially for positive $p^{2}$. Does it mean that this kind of model is not valid from the point of view of quantum field theory [34]? We cannot immediately conclude that such types of propagators cannot be considered because around the nonlocality scale the model undergoes a strong coupling regime which means that oneloop approximation is not valid anymore. However, this

\footnotetext{
${ }^{4}$ The curves also grow for small momenta, reproducing the logarithmic divergence in the local limit but this growing is not apparent for the chosen domain of the numeric integration.
} 


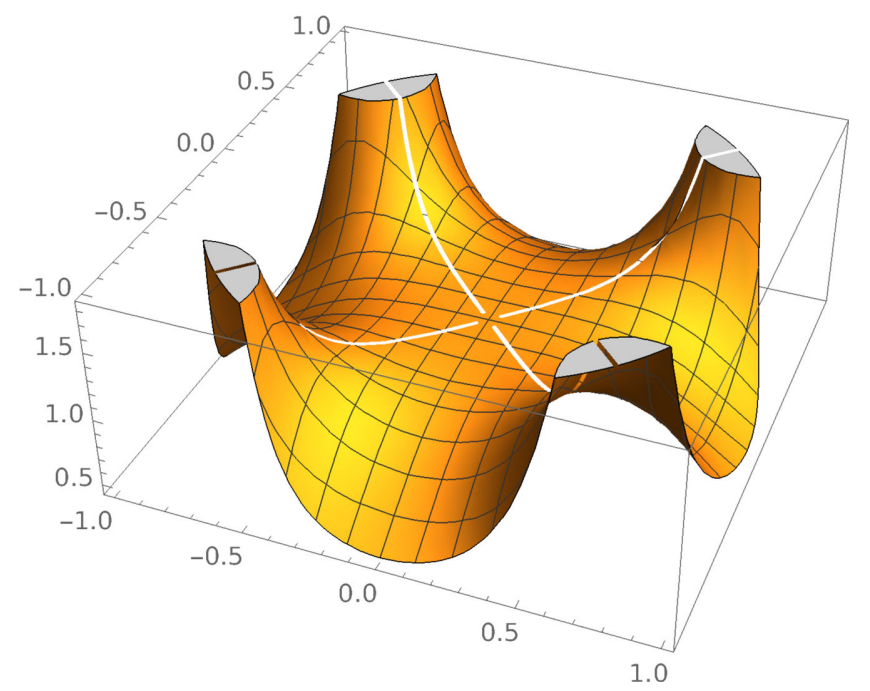

FIG. 5. Absolute value of the function $f_{2}(\operatorname{Re} k, \operatorname{Im} k)$.

does not mean that the unitarity is broken as long as nonperturbative methods can, in principle, lead to a result which is still consistent with unitarity. We leave this question for future study.

In this work we concentrate on the results which can be obtained perturbatively. If the propagator $f\left(k^{2}\right) / k^{2}$ is falling for both signs of $k^{2}$ then we expect the loop contributions to fall for all physical values of external momenta. However, the scattering amplitude would inevitably grow for some directions of $s$ and $t$ in the complex plane. Let us check the consistency of such a behavior with the known results about the properties of the amplitudes. For example, the Martin-Froissart bound [35] tells us that the amplitude in the forward limit $t \rightarrow 0$ is bounded by

$$
|\mathcal{M}(s, 0)|<C s(\log s)^{2}
$$

on the $s$ complex plane. Although this bound was obtained in local theories, the recent work [34] provides a formal proof of the similar bound in nonlocal theories,

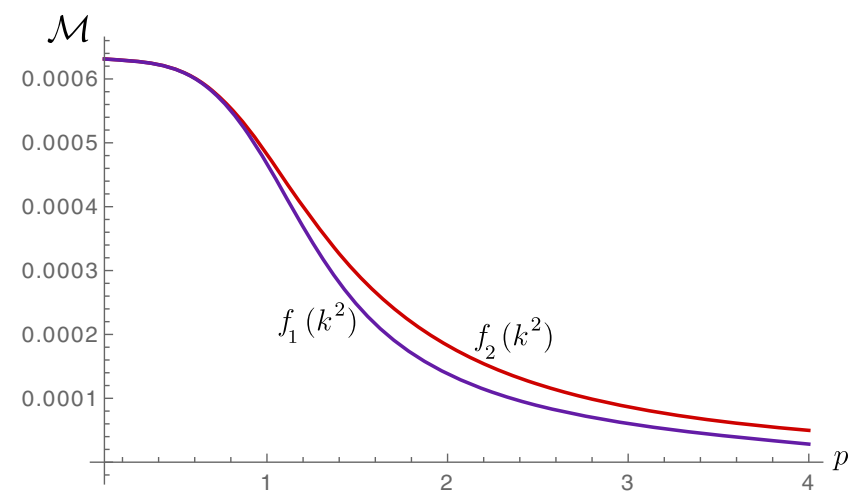

FIG. 7. Scattering 2-2 one-loop amplitude as a function of the external momentum. The red curve is for form-factor $f_{1}(k)$ and blue curve is for form-factor $f_{2}(k)$. Here we have taken $\lambda=0.1$.

$$
|\mathcal{M}(s, 0)| \lesssim C s^{1+2 a} .
$$

This bound was obtained within rather weak assumptions including the validity of partial wave expansion, unitarity, analyticity, and the exponential boundedness $|\mathcal{M}(s, t)|<$ $s^{N} e^{a|s|^{a}}$ for fixed $t$. Here $\alpha, N, C$ are some positive constants. These assumptions, except the last one, are expected to be fulfilled in the models we consider in this work and in more generic settings like in [26]. The bound (38) actually means that the amplitudes in the forward limit are polynomially bounded while one would expect the exponential growth in some directions on the complex $s$ plane.

Let us discuss this issue using the simple model with a cubic interaction where the exponential behavior shows up already at tree level.

$$
S=\int d^{4} x\left[\frac{1}{2} \phi\left(\square-m^{2}\right) f(\square)^{-1} \phi-\frac{\lambda}{3 !} \phi^{3}\right]
$$

The tree level amplitude in this model has the form
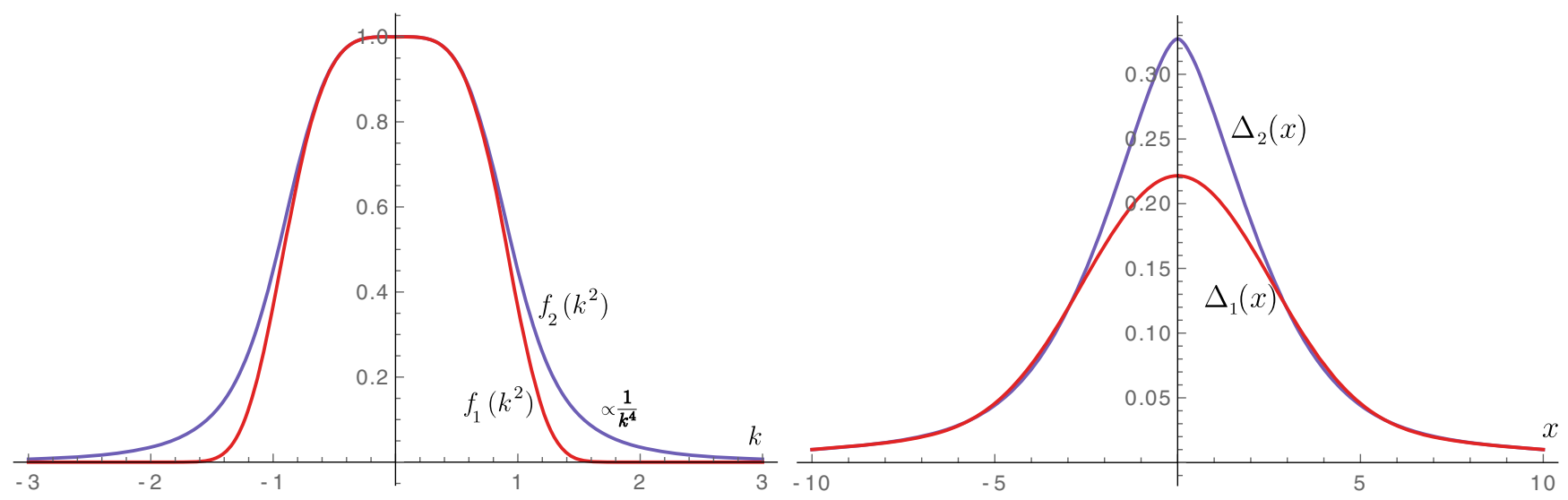

FIG. 6. The left plot represents the functions $f_{1}(k), f_{2}(k)$ and the right plot shows their Fourier images $\Delta_{1,2}(x)$. 


$$
\mathcal{M}(s, t) \sim \lambda^{2}\left(\frac{f(s)}{s-m^{2}}+\frac{f(t)}{t-m^{2}}+\frac{f(u)}{u-m^{2}}\right) .
$$

Thus, $f(s)$ must have a direction of exponential growth since an entire function, which is polynomially bounded on the whole complex plane, is nothing else but a polynomial of finite order. However, for all physical momenta the amplitude is small if we require $f\left(q^{2}\right)$ to fall for real $q^{2}$. Thus, with this choice, the model stays in the perturbative regime for physical values of momenta. However, if one computes the amplitude for complex values of momenta this could lead to breaking of the perturbation theory. The naive computation leading to the exponential growth can be invalid. Therefore, the conclusion about the amplitude's exponential growth in the nonphysical region is not correct.

The bound (38) leads to further interesting consequences for the model (39). Under the condition of polynomial boundedness of the amplitude in the forward limit, we have the Cerulus-Martin lower bound on the amplitude falling [36],

$$
|\mathcal{M}(s, t \rightarrow 0)|>C e^{-\gamma \sqrt{s} \log \left(s / s_{0}\right)} .
$$

Here $C, \gamma, s_{0}$ are positive constants. This bound is obviously violated if we take $f\left(k^{2}\right) \propto e^{-\alpha k^{4}}$. The tree level amplitude is falling too fast while the model is in a perturbative regime so we can trust this computation for physical momenta. This contradiction means that something is wrong with the model with an exponentially falling propagator. Notice that in the work [8] the choice of polynomially suppressed propagators was suggested to be motivated by different arguments such as power counting, for example. The propagator falling as $1 / k^{n}$ is still consistent with the discussed bounds.

Notice that disfavoring the exponentially suppressed propagator is not applicable for models with contact $\phi^{4}$ interactions since the leading term is given by a constant tree level contribution. This kind of suppression for those models is not in a contradiction with Martin-Cerulus bound [36].

\section{CONCLUSION AND OUTLOOK}

We have shown explicitly that the optical theorem statement holds in nonlocal higher-derivative theories upon utilizing a modified computation prescription. The prescription consists of computing Feynman graphs, assuming all the internal momenta are Euclidean and then continuing the final answer analytically to Minkowski signature for external momenta. Resulting expressions satisfy the optical theorem statement and obey the correct local theory limit. We were able to show that the one-loop correction to the vertex in a nonlocal $\phi^{4}$ theory can be computed for arbitrary form factors and have presented both analytic and numeric derivations of the result. We found that, independent of the nonlocal form factor, the leading term in the amplitude at high energies falls as $\sim 1 / p^{2}$ as long as $f\left(k^{2}\right)=f\left(-k^{2}\right)$. This term arises as a result of the computation of the pole contribution to the amplitude. Thus, nonlocal amplitudes for even form factors at one loop have a universal behavior in the UV limit.

As an important observation we have found that under an assumption that the Martin-Cerulus bound [36] is as satisfied in nonlocal models as in local ones, then the amplitudes should not fall too fast obeying bound (41). If such a nonlocal theory has a contact interaction then this bound can always be satisfied irrespectively of the decay rate of the propagator as the contact interaction term always dominates in the perturbative expansion. On the other hand, in the absence of the contact interaction (for instance computing corrections to 2-2 scattering in $\phi^{3}$ theory) one must demand that propagator decays polynomially favoring as such the class of functions suggested in [8].

As the next step, one would aim at demonstrating that the background field method provides consistent results and thus can be applied to AID models which is a plausible expectation. Also the strong coupling regime, which may arise in such nonlocal field theories [20] due to the growth of the amplitudes for complex parameters, is an important question for further investigations. Yet another ambitious problem is to study such theories in the large- $N$ approximations [37] and see how they are compared to their local counterparts.

\section{ACKNOWLEDGMENTS}

The authors would like to thank I. Aref'eva and M. Shaposhnikov for comments and questions on the manuscript. A. K. is supported by FCT Portugal investigator Project No. IF/01607/2015. A. T. is supported by the Academy of Finland Grant No. 318319. The part of the work performed by A. T. related to the numeric computations of the amplitudes is supported by the Russian Science Foundation Grant No. 19-12-00393.

Note added.-Upon completion of the manuscript we have met with great interest a related study [38] discussing the same problem of unitarity of nonlocal field theories. 
[1] M. Ostrogradsky, Memoires sur les equations differentielles relatives au probleme des isoperimetres, Mem. Ac. St. Petersbourg VI, 385 (1850).

[2] G. V. Efimov, Non-local quantum theory of the scalar field, Commun. Math. Phys. 5, 42 (1967).

[3] S. Coleman, Resonance poles and resonance multipoles, in Theory and Phenomenology in Particle Physics, Part B, edited by A. Zichichi (Academic Press, New York, 1969), pp. 659-683.

[4] K. S. Stelle, Renormalization of higher derivative quantum gravity, Phys. Rev. D 16, 953 (1977).

[5] E.S. Fradkin and A. A. Tseytlin, Renormalizable asymptotically free quantum theory of gravity, Phys. Lett. 104B, 377 (1981).

[6] Y. Kuzmin, The convergent nonlocal gravitation (In Russian), Sov. J. Nucl. Phys. 50, 1011 (1989).

[7] N. Krasnikov, Nonlocal gauge theories, Theor. Math. Phys. 73, 1184 (1987).

[8] E. Tomboulis, Superrenormalizable gauge and gravitational theories, arXiv:hep-th/9702146.

[9] L. Brekke, P. G. Freund, M. Olson, and E. Witten, Nonarchimedean string dynamics, Nucl. Phys. B302, 365 (1988).

[10] V. Vladimirov, I. Volovich, and E. Zelenov, p-Adic analysis and mathematical physics, Series on Soviet and East European mathematics, 1, (World Scientific, Singapore, 1994), pp. 1-319.

[11] B. Dragovich, A. Y. Khrennikov, S. Kozyrev, I. Volovich, and E. Zelenov, $p$-Adic mathematical physics: The first 30 years, Anal. Appl. 9, 87 (2017).

[12] D. Anselmi, Fakeons and Lee-Wick models, J. High Energy Phys. 02 (2018) 141.

[13] A. Nicolis, R. Rattazzi, and E. Trincherini, The Galileon as a local modification of gravity, Phys. Rev. D 79, 064036 (2009).

[14] G. W. Horndeski, Second-order scalar-tensor field equations in a four-dimensional space, Int. J. Theor. Phys. 10, 363 (1974).

[15] T. Kobayashi, Horndeski theory and beyond: A review, Rep. Prog. Phys. 82, 086901 (2019).

[16] T. Biswas, E. Gerwick, T. Koivisto, and A. Mazumdar, Towards Singularity and Ghost Free Theories of Gravity, Phys. Rev. Lett. 108, 031101 (2012).

[17] T. Biswas, A. S. Koshelev, and A. Mazumdar, Consistent higher derivative gravitational theories with stable de Sitter and anti-de Sitter backgrounds, Phys. Rev. D 95, 043533 (2017).

[18] L. Keltner and A. J. Tolley, UV properties of Galileons: Spectral densities, arXiv:1502.05706.

[19] A. S. Koshelev, K. S. Kumar, and A. A. Starobinsky, Analytic infinite derivative gravity, $R^{2}$-like inflation, quantum gravity and CMB, Int. J. Mod. Phys. D 29, 2043018 (2020).
[20] A. S. Koshelev and A. Tokareva, Nonlocal self-healing of Higgs inflation, Phys. Rev. D 102, 123518 (2020).

[21] J. Boos, V. P. Frolov, and A. Zelnikov, Ghost-free modification of the Polyakov action and Hawking radiation, Phys. Rev. D 100, 104008 (2019).

[22] E. Witten, Noncommutative geometry and string field theory, Nucl. Phys. B268, 253 (1986).

[23] E. Witten, Interacting field theory of open superstrings, Nucl. Phys. B276, 291 (1986).

[24] K. Ohmori, A review on tachyon condensation in open string field theories, other thesis, 2001.

[25] I. Arefeva, D. Belov, A. Giryavets, A. Koshelev, and P. Medvedev, Noncommutative field theories and (super)string field theories, in 11th Jorge Andre Swieca Summer School on Particle and Fields (2001), Vol. 11, pp. 1-163 [arXiv: hep-th/0111208].

[26] R. Pius and A. Sen, Cutkosky rules for superstring field theory, J. High Energy Phys. 10 (2016) 024; Erratum, J. High Energy Phys. 09 (2018) 122.

[27] M. E. Peskin and D. V. Schroeder, An Introduction to Quantum Field Theory (Addison-Wesley, Reading, USA, 1995).

[28] R. Pius and A. Sen, Unitarity of the box diagram, J. High Energy Phys. 11 (2018) 094.

[29] F. Briscese and L. Modesto, Cutkosky rules and perturbative unitarity in Euclidean nonlocal quantum field theories, Phys. Rev. D 99, 104043 (2019).

[30] L. Buoninfante, G. Lambiase, and A. Mazumdar, Ghost-free infinite derivative quantum field theory, Nucl. Phys. B944, 114646 (2019).

[31] I. S. Gradshteyn and I. M. Ryzhik, Table of Integrals, Series, and Products (Elsevier/Academic Press, Amsterdam, Amsterdam, The Netherlands, 2007).

[32] W. N. Bailey, Some infinite integrals involving bessel functions, Proc. London Math. Soc. s2-40, 37 (1936).

[33] P. Chin and E. T. Tomboulis, Nonlocal vertices and analyticity: Landau equations and general Cutkosky rule, J. High Energy Phys. 06 (2018) 014.

[34] J. Tokuda, Extension of positivity bounds to non-local theories: IR obstructions to Lorentz invariant UV completions, J. High Energy Phys. 05 (2019) 216.

[35] A. Martin, Extension of the axiomatic analyticity domain of scattering amplitudes by unitarity. 1, Nuovo Cimento A 42, 930 (1966).

[36] F. A. Cerulus and A. Martin, A lower bound for largeangle elastic scattering at high energies, Phys. Lett. 8, 80 (1964).

[37] G. 't Hooft; Large N, in The Phenomenology of Large N(c) QCD (2002), Vol. 4, pp. 3-18 [arXiv:hep-th/0204069].

[38] F. Briscese and L. Modesto, Non-unitarity of Minkowskian non-local quantum field theories, arXiv:2103.00353. 